\title{
Witt's theorem in abstract geometric algebra
}

\author{
Patrice P. Ntumba \\ Department of Mathematics and Applied Mathematics, \\ University of Pretoria, Hatfield 0002, Pretoria, Republic of South Africa \\ e-mail: patrice.ntumba@up.ac.za
}

\begin{abstract}
In an earlier paper of the author, a version of the Witt's theorem was obtained within a specific subcategory of the category of $A$-modules: the full subcat-egory of convenient A-modules. A further investigation yields two more versions of the Witt's theorem by revising the notion of convenient $A$-modules. For the first version, the $A$-bilinear form involved is either symmetric or antisymmetric, and the two isometric free sub- $A$-modules, the isometry between which may extend to an isom-etry of the non-isotropic convenient $A$-module concerned onto itself, are assumed pre-hyperbolic. On the other hand, for the second version, the $A$-bilinear form defined on the non-isotropic convenient $A$-module involved is set to be symmetric, and the two isometric free sub- $A$-modules, whose orthogonals are to be proved isometric, are assumed strongly non-isotropic and disjoint.
\end{abstract}

Keywords Sheaf of $A$-radicals - Orthosymmetric $A$-bilinear forms ·

Strongly isotropic (non-isotropic) sub- $A$-modules $\cdot$ Weakly isotropic (non-isotropic) sub- $A$-modules $\cdot$ Free subpresheaves of modules ·

Pre-hyperbolic free sub- $A$-modules 


\section{Basics of abstract geometric algebra}

As has been said by Mallios in many of his works, we would cite here for example [712], and [13], Abstract Differential Geometry (ADG in short) offers a new approach to classical Differential Geometry, in the sense that obstacles, which do appear when trying to cope with problems of quantum gravity, within the standard set-up of the classical Differential Geometry, do not appear at all within the context of ADG. The spirit of ADG is to perform differential geometry, no space (viz. smooth manifolds) is virtually required, provided that one has at their disposal a basic differential, $\partial$, alias $d x$, along with the appropriate differential-geometric mechanism that might be afforded thereby.

The major goal of this paper consists, as indicated in the title, in the setting of the sheaf-theoretic version of the Witt's theorem. The classical Witt's theorem has several versions, see to this effect for instance [1-5], and [6]. Our main reference as far as abstract geometric algebra is concerned is Mallios[7].

This work is meant to be part of the ongoing project as undertaken in MalliosNtumba [14-16], and Ntumba-Orioha [18].

All our $\mathcal{A}$-modules and $A$-presheaves in this paper are defined on a fixed topological space $X$ and are torsion-free. $\mathcal{A}$-modules and $A$-presheaves with their respective morphisms form categories which we denote $\mathcal{A}-\mathcal{M} \operatorname{Md}_{X}$ and $A-\mathcal{P} \mathcal{S} h_{X}$, respectively. By virtue of the equivalence $\mathcal{S} h_{X} \cong \mathcal{C} o \mathcal{P S} h_{X}$, an $\mathcal{A}$-morphism $\phi: \mathcal{E} \longrightarrow \mathcal{F}$ of $\mathcal{A}$-modules $\mathcal{E}$ and $\mathcal{F}$ may be identified with the $A$-morphism $\bar{\phi}:=\left(\bar{\phi}_{U}\right)_{X \supseteq U \text {, open }}: E \longrightarrow F$ of the associated $A$-presheaves. We shall most often denote by just $\phi$ the corresponding $A$-morphism associated with the $\mathcal{A}$-morphism $\phi$. The meaning of $\phi$ will always be determined by the situation at hand.

Recall that given an $\mathcal{A}$-module $\mathcal{E}$ and a sub- $\mathcal{A}$-module $\mathcal{F}$ of $\mathcal{E}$, the quotient $\mathcal{A}$-module of $\mathcal{E}$ by $\mathcal{F}$ is the $\mathcal{A}$-module generated by the presheaf sending an open $U \subseteq X$ to an $\mathcal{A}(U)$-module $S(U):=\Gamma(U, \mathcal{E}) / \Gamma(U, \mathcal{F}) \equiv \mathcal{E}(U) / \mathcal{F}(U)$ such that for every restriction map $\sigma_{V}^{U}: \mathcal{E}(U) / \mathcal{F}(U) \longrightarrow \mathcal{E}(V) / \mathcal{F}(V)$, one has $\sigma_{V}^{U}(r+\mathcal{F}(U)):=$ $\rho_{V}^{U}(r)+\mathcal{F}(V)$ (the $\rho_{V}^{U}$ are the restriction maps for the $A$-presheaf $\Gamma \mathcal{E}$ ).

For the sake of easy referencing, we also recall some notions, which may be found in our recent papers such as [14-16], and [18]. Let $\mathcal{E}$ and $\mathcal{F}$ be $\mathcal{A}$-modules and $\phi: \mathcal{E} \oplus \mathcal{F} \longrightarrow \mathcal{A}$ an $\mathcal{A}$-bilinear morphism. Then, we say that the triple $(\mathcal{E}, \mathcal{F} ; \phi)$ forms a pairing of $\mathcal{A}$-modules or that $\mathcal{E}$ and $\mathcal{F}$ are paired through $\phi$ into $\mathcal{A}$. The sub- $\mathcal{A}$-module $\mathcal{F}^{\perp}$ of $\mathcal{E}$ such that, for every open subset $U$ of $X, \mathcal{F}^{\perp}(U)$ consists of all $r \in \mathcal{E}(U)$ with $\phi_{V}\left(\mathcal{F}(V),\left.r\right|_{V}\right)=0$ for any open $V \subseteq U$, is called the left kernel of the pairing $(\mathcal{E}, \mathcal{F} ; \phi)$. In a similar way, one defines the right kernel of $(\mathcal{E}, \mathcal{F} ; \phi)$ to be the sub- $\mathcal{A}$-module $\mathcal{E}^{\perp}$ of $\mathcal{F}$ such that, for any open subset $U$ of $X$, $\mathcal{E}^{\perp}(U)$ is the set of all (local) sections $r \in \mathcal{F}(U) \operatorname{such} \phi_{V}\left(\left.r\right|_{V}, \mathcal{E}(V)\right)=0$ for every open $V \subseteq U$. If $(\mathcal{E}, \mathcal{F} ; \phi)$ is a pairing of free $\mathcal{A}$-modules, then, for every open subset $U$ of $X, \mathcal{F}^{\perp}(U)=\mathcal{F}(U)^{\perp}:=\left\{r \in \mathcal{E}(U): \phi_{U}(\mathcal{F}(U), r)=0\right\}$, and similarly $\mathcal{E}^{\perp}(U)=\mathcal{E}(U)^{\perp}:=\left\{r \in \mathcal{F}(U): \phi_{U}(r, \mathcal{E}(U))=0\right\}$. Let us recall at this stage that the dual of $\mathcal{E}$ is denoted $\mathcal{E}^{*}$, see [7, p. 298]. A pairing $(\mathcal{E}, \mathcal{F} ; \phi)$ of free $\mathcal{A}$-modules is called a weakly convenient pairing if given free sub- $\mathcal{A}$-modules $\mathcal{F}_{0}$ and $\mathcal{E}_{0}$ of $\mathcal{F}$ and $\mathcal{E}$, respectively, their orthogonal $\mathcal{F}_{0}^{\perp}$ and $\mathcal{E}_{0}^{\perp}$ are free sub- $\mathcal{A}$-modules of $\mathcal{E}$ and $\mathcal{F}$, respectively. 
Now, let $(\mathcal{E}, \mathcal{E} ; \phi)$ be a pairing such that if $r, s \in \mathcal{E}(U)$, where $U$ is an open subset of $X$, then $\phi_{U}(r, s)=0$ if and only if $\phi_{U}(s, r)=0$. The left kernel, $\mathcal{E}_{l}^{\perp}:=\mathcal{E}^{\perp}$, is the same as the right kernel $\mathcal{E}_{r}^{\perp}:=\mathcal{E}^{\top}$. In this instance, we say that the $\mathcal{A}$-bilinear form $\phi$ is orthosymmetric and call $\mathcal{E}^{\perp}\left(=\mathcal{E}^{\top}\right)$ the radical sheaf (or sheaf of $\mathcal{A}$-radicals, or simply $\mathcal{A}$-radical) of $\mathcal{E}$, and denote it by $\operatorname{rad}_{\mathcal{A}} \mathcal{E} \equiv \operatorname{rad} \mathcal{E}$. An $\mathcal{A}$-module $\mathcal{E}$ such that $\operatorname{rad} \mathcal{E} \neq 0$ (resp. $\operatorname{rad} \mathcal{E}=0$ ) is called isotropic (resp. non-isotropic); $\mathcal{E}$ is totally isotropic if $\phi$ is identically zero, i.e. $\phi_{U}(r, s)=0$ for all sections $r, s \in \mathcal{E}(U)$, with $U$ open in $X$. For any open $U \subseteq X$, a non-zero section $r \in \mathcal{E}(U)$ is called isotropic if $\phi_{U}(r, r)=0$. For a sub- $\mathcal{A}$-module $\mathcal{F}$ of $\mathcal{E}$, the $\mathcal{A}$-radical of $\mathcal{F}$ is defined as rad $\mathcal{F}:=\mathcal{F} \cap \mathcal{F}^{\perp}=\mathcal{F} \cap \mathcal{F}^{\top}$. If $\operatorname{rad} \mathcal{F}=0$, then $\mathcal{F}$ is said to be strongly non-isotropic. In other words, for every section $r \in \mathcal{F}(U)$, there exists a section $s \in \mathcal{F}(U)$ such that $\phi_{U}(r, s) \neq 0$. If $(\operatorname{rad} \mathcal{F})(U) \neq 0$ for every open $U \subseteq X$, then $\mathcal{F}$ is said to be strongly isotropic. However, it is possible that a sub- $\mathcal{A}$-module $\mathcal{F}$ is neither strongly isotropic, nor strongly non-isotropic; in such a case, $\mathcal{F}$ is said to either be weakly isotropic or weakly non-isotropic. Now, let $(\mathcal{E}, \mathcal{F} ; \phi)$ be a pairing of free $\mathcal{A}$-modules, then for every open subset $U$ of $X,(\operatorname{rad} \mathcal{E})(U)=\operatorname{rad} \mathcal{E}(U)$ and $(\operatorname{rad} \mathcal{F})(U)=\operatorname{rad} \mathcal{F}(U)$, where $\operatorname{rad} \mathcal{E}(U)=\mathcal{E}(U) \cap \mathcal{E}(U)^{\perp}$ and $\operatorname{rad} \mathcal{F}(U)=\mathcal{F}(U) \cap \mathcal{F}(U)^{\perp}$. Given a pairing $(\mathcal{E}, \mathcal{E} ; \phi)$ with $\phi$ a symmetric $\mathcal{A}$-bilinear morphism, sub- $\mathcal{A}$-modules $\mathcal{E}_{1}$ and $\mathcal{E}_{2}$ of $\mathcal{E}$ are said to be mutually orthogonal if for every open subset $U$ of $X, \phi_{U}(r, s)=0$, for all $r \in \mathcal{E}_{1}(U)$ and $s \in \mathcal{E}_{2}(U)$. If $\mathcal{E}=\oplus_{i \in I} \mathcal{E}_{i}$, where the $\mathcal{E}_{i}$ are pairwise orthogonal sub- $\mathcal{A}$-modules of $\mathcal{E}$, we say that $\mathcal{E}$ is the direct orthogonal sum of the $\mathcal{E}_{i}$, and write $\mathcal{E}:=\mathcal{E}_{1} \perp \cdots \perp \mathcal{E}_{i} \perp \cdots$

Lemma 1 Let $\phi$ be a non-degenerate $\mathcal{A}$-bilinear form on an $\mathcal{A}$-module $\mathcal{E}$. Then the mappings $\perp \equiv \perp(\phi), \top \equiv \top(\phi)$ have the following properties:

(1) (a) If $\mathcal{G} \subseteq \mathcal{H}$, then $\mathcal{G}^{\perp} \supseteq \mathcal{H}^{\perp}$

(b) If $\mathcal{G} \subseteq \mathcal{H}$, then $\mathcal{G}^{\top} \supseteq \mathcal{H}^{\top}$

(c) $(\mathcal{G}+\mathcal{H})^{\perp}=\mathcal{G}^{\perp} \cap \mathcal{H}^{\perp}$

(d) $(\mathcal{G}+\mathcal{H})^{\top}=\mathcal{G}^{\top} \cap \mathcal{H}^{\top}$

for all sub-A-modules $\mathcal{G}$ and $\mathcal{H}$ of $\mathcal{E}$.

Proof Assertion (1) is clear. However, to prove (2), one has to take care of the very definition of the bi-functor $\mathcal{H o m}_{\mathcal{A}}(\cdot, \cdot)$ since it concerns the operation " $\perp$ ": thus, one should consider the sheaves (: $\mathcal{A}$-modules) involved as (complete) presheaves (à la Leray) to handle the corresponding morphisms; see e.g. [7, p. 133, (6.4)/(6.5)].

Now for the sake of what follows, we assume, unless otherwise mentioned, that the pair $(X, \mathcal{A})$ is an algebraized space, where $\mathcal{A}$ is a unital $\mathbb{C}$-algebra sheaf such that every nowhere-zero section of $\mathcal{A}$ is invertible.

Theorem 1, which has been proved in [15], is pivotal as far as the sheaf-theoretic version of the Witt's theorem is concerned.

Theorem 1 Let $(\mathcal{E}, \phi)$ be a free $\mathcal{A}$-module of finite rank. Then, every non-isotropic free sub-A-module $\mathcal{F}$ of $\mathcal{E}$ is a direct summand of $\mathcal{E}$; viz.

$$
\mathcal{E}=\mathcal{F} \perp \mathcal{F}^{\perp}
$$


Next, let us revise the notion of convenient $\mathcal{A}$-modules by altering its format of [15] so as to make some of its hypotheses redundant in case the coefficient algebra sheaf $\mathcal{A}$ is a PID algebra sheaf (i.e., for every open $U \subseteq X$, the algebra $\mathcal{A}(U)$ is a PID algebra; in other words, given a free $\mathcal{A}$-module and a sub- $\mathcal{A}$-module $\mathcal{F} \subseteq \mathcal{E}$, one has that $\mathcal{F}$ is section-wise free), see [17].

To this end, we need the following

Definition 1 (A. Mallios) A subpresheaf $F$ of a presheaf of modules (or more precisely, $A(U)$-modules) $E$ (cf. [7, p. 99, Definition 1.6]) is called a free subpresheaf if for every open $U$ in $X, F(U)$ is a free sub- $A(U)$-module of $E(U)$.

Then, we have

Definition 2 A convenient $\mathcal{A}$-module is a self pairing $(\mathcal{E}, \mathcal{E} ; \phi) \equiv(\mathcal{E}, \phi)$, where $\mathcal{E}$ is a free $\mathcal{A}$-module of finite rank and $\phi$ an orthosymmetric $\mathcal{A}$-bilinear form, such that the following conditions are satisfied: (1) If $\mathcal{F}$ is a free subpresheaf of $\mathcal{A}(U)$-modules of $\mathcal{E}$, then $\mathcal{F}^{\perp} \equiv \mathcal{F}^{\perp_{\phi}}$ is a free subpresheaf of $\mathcal{A}(U)$-modules of $\mathcal{E}$; (2) Every free subpresheaf $\mathcal{F}$ of $\mathcal{A}(U)$-modules of $\mathcal{E}$ is orthogonally reflexive, i.e., $\mathcal{F}^{\perp \perp}=\mathcal{F}$; (3) The intersection of any two free subpresheaves of $\mathcal{A}(U)$-modules of $\mathcal{E}$ is a free subpresheaf of $\mathcal{A}(U)$-modules.

Note Concerning the above definition of convenient $\mathcal{A}$-modules, by supposing that the (coefficient-) algebra sheaf $\mathcal{A}$ is a PID-algebra sheaf, we obtain that every subpresheaf of $\mathcal{A}(U)$-modules of a free $\mathcal{A}$-module is free. So in that context, conditions (1) and (3) in Definition 5 are satisfied. Now, concerning condition (2) of the same definition, the reflexivity at hand is a known situation in ordinary Functional Analysis: see, for instance, Hilbert spaces and structures having similar properties; so we do have the so-called complemented topological algebras, Hilbert algebras and the likes with the aforementioned property for ideals (: modules), and also analogous examples in infinite-dimensional Hamiltonian mechanics. (I am indebted to A. Mallios for this comment on convenient $\mathcal{A}$-modules.)

Orthogonally convenient pairings of $\mathcal{A}$-modules are just as much interesting as convenient $\mathcal{A}$-modules and satisfy in some restricted way conditions (1) and (2) of Definition 5.

Definition 3 A pairing $(\mathcal{E}, \mathcal{F} ; \phi)$ of free $\mathcal{A}$-modules $\mathcal{E}$ and $\mathcal{F}$ into the $\mathbb{C}$-algebra sheaf $\mathcal{A}$ is called an orthogonally convenient pairing if given free sub- $\mathcal{A}$-modules $\mathcal{E}_{0}$ and $\mathcal{F}_{0}$ of $\mathcal{E}$ and $\mathcal{F}$, respectively, their orthogonal $\mathcal{E}_{0}^{\perp}$ and $\mathcal{F}_{0}^{\perp}$ are free sub- $\mathcal{A}$-modules of $\mathcal{F}$ and $\mathcal{E}$, respectively.

Based on [14, pp. 399-401], if $\mathcal{E}$ is an $\mathcal{A}$-module, $\mathcal{F}$ and $\mathcal{G}$ are sub- $\mathcal{A}$-modules of $\mathcal{E}$ such $\mathcal{E}=\mathcal{F} \oplus \mathcal{G}$, then

$$
\mathcal{E} / \mathcal{F}=\mathcal{G}
$$

within an $\mathcal{A}$-isomorphism. Furthermore, if $\mathcal{E}$ is a free $\mathcal{A}$-module and $\mathcal{F}$ a free sub- $\mathcal{A}$ module of $\mathcal{E}$, then, for every open $U \subseteq X$, 
On another side, given an orthogonally convenient $\mathcal{A}$-pairing $(\mathcal{E}, \mathcal{F} ; \phi), \mathcal{E}_{0}$ and $\mathcal{F}_{0}$ free sub- $\mathcal{A}$-modules of (free $\mathcal{A}$-modules) $\mathcal{E}$ and $\mathcal{F}$, respectively, one has, for every open $U \subseteq X$,

$$
\left(\mathcal{E} / \mathcal{F}_{0}^{\perp}\right)(U)=\mathcal{E}(U) / \mathcal{F}_{0}^{\perp}(U)
$$

and

$$
\left(\mathcal{F} / \mathcal{E}_{0}^{\perp}\right)(U)=\mathcal{F}(U) / \mathcal{E}_{0}^{\perp}(U)
$$

From [14], we also single out the following result.

Theorem 2 Let $\mathcal{E}$ and $\mathcal{F}$ be $\mathcal{A}$-modules paired into a $\mathbb{C}$-algebra sheaf $\mathcal{A}$, and assume

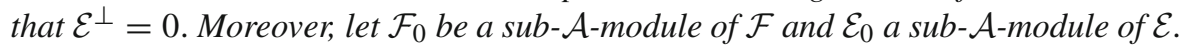
There exist natural $\mathcal{A}$-isomorphisms into:

$$
\mathcal{E} / \mathcal{F}_{0}^{\perp} \longrightarrow \mathcal{F}_{0}^{*}
$$

and

$$
\mathcal{E}_{0}^{\perp} \longrightarrow\left(\mathcal{E} / \mathcal{E}_{0}\right)^{*}
$$

Definition 4 The pairing $\left(\mathcal{E}, \mathcal{E}^{*} ; \phi\right)$, where $\mathcal{E}$ is a free $\mathcal{A}$-module and such that for every open $U \subseteq X$,

$$
\phi_{U}(r, \psi):=\psi_{U}(r)
$$

with $\psi \in \mathcal{E}^{*}(U):=\operatorname{Hom}_{\left.\mathcal{A}\right|_{U}}\left(\left.\mathcal{E}\right|_{U},\left.\mathcal{A}\right|_{U}\right)$ and $r \in \mathcal{E}(U)$, is called the canonical pairing of $\mathcal{E}$ and $\mathcal{E}^{*}$.

Theorem 3 Let $\mathcal{E}$ be a free $\mathcal{A}$-module of finite rank. The canonical pairing $\left(\mathcal{E}, \mathcal{E}^{*} ; \phi\right)$ is orthogonally convenient.

Proof First, we notice by [14, Theorem 2.2] that both kernels, i.e. $\mathcal{E}^{\perp}$ and $\left(\mathcal{E}^{*}\right)^{\perp}$, are 0 . Let $\mathcal{E}_{0}$ be a free sub- $\mathcal{A}$-module of $\mathcal{E}$, and consider the map (6) of Theorem 2: $\mathcal{E}_{0}^{\perp} \longrightarrow\left(\mathcal{E} / \mathcal{E}_{0}\right)^{*}$. It is an $\mathcal{A}$-isomorphism into, and we shall show that it is onto. Fix an open set $U$ in $X$, and let $\psi \in\left(\mathcal{E} / \mathcal{E}_{0}\right)^{*}(U):=\operatorname{Hom}_{\left.\mathcal{A}\right|_{U}}\left(\left.\left(\mathcal{E} / \mathcal{E}_{0}\right)\right|_{U},\left.\mathcal{A}\right|_{U}\right)$. Let us consider a family $\bar{\psi} \equiv\left(\bar{\psi}_{V}\right)_{U \supseteq V \text {, open }}$ where if $V, W$ are open in $U$ with $W \subseteq V$, then

$$
\tau_{W}^{V} \circ \bar{\psi}_{V}=\bar{\psi}_{W} \circ \rho_{W}^{V}
$$

(the $\left\{\rho_{V}^{U}\right\}$ and $\left\{\tau_{V}^{U}\right\}$ are the restriction maps for the (complete) presheaf of sections of $\mathcal{E}$ and $\mathcal{A}$, respectively) and

$$
\bar{\psi}_{V}(r):=\psi_{V}\left(r+\mathcal{E}_{0}(V)\right), r \in \mathcal{E}(V) .
$$


It is easy to see that $\bar{\psi}_{V}$ is $\mathcal{A}(V)$-linear for any open $V \subseteq U$. Thus,

$$
\bar{\psi} \in \operatorname{Hom}_{\left.\mathcal{A}\right|_{U}}\left(\left.\mathcal{E}\right|_{U},\left.\mathcal{A}\right|_{U}\right)=: \mathcal{E}^{*}(U)
$$

Suppose $r \in \mathcal{E}_{0}(V)$, where $V$ is open in $U$. Then

$$
\bar{\psi}_{V}(r)=\psi_{V}\left(r+\mathcal{E}_{0}(V)\right)=\psi_{V}\left(\mathcal{E}_{0}(V)\right)=0,
$$

therefore

$$
\phi_{V}\left(\mathcal{E}_{0}(V),\left.\bar{\psi}\right|_{V}\right)=\bar{\psi}_{V}\left(\mathcal{E}_{0}(V)\right)=0
$$

i.e. $\bar{\psi} \in \mathcal{E}_{0}^{\perp}(U)$. We contend that $\bar{\psi}$ has the given $\psi$ as image under the map (6), and this will show the ontoness of (6) and that $\mathcal{E}_{0}^{\perp}$ is a free sub- $\mathcal{A}$-module of $\mathcal{E}^{*}$.

Let us find the image of $\bar{\psi}$. Consider the pairing $\left(\mathcal{E} / \mathcal{E}_{0}, \mathcal{E}_{0}^{\perp} ; \Theta\right)$ such that for any open $V \subseteq X$, we have

$$
\Theta_{V}\left(r+\mathcal{E}_{0}(V), \alpha\right):=\phi_{V}(r, \alpha)=\alpha_{V}(r),
$$

where $r \in \mathcal{E}(V), \alpha \in \mathcal{E}_{0}^{\perp}(V) \subseteq \mathcal{E}^{*}(V)$. Clearly, the right kernel of this new pairing is 0 . For $\alpha=\bar{\psi} \in \mathcal{E}_{0}^{\perp}(U) \subseteq \mathcal{E}^{*}(U)$, we have

$$
\Theta_{U}\left(r+\mathcal{E}_{0}(U), \bar{\psi}\right)=\bar{\psi}_{U}(r)
$$

where $r \in \mathcal{E}(U)$, and the map

$$
\bar{\Theta}_{U}: \mathcal{E}_{0}^{\perp}(U) \longrightarrow\left(\mathcal{E} / \mathcal{E}_{0}\right)^{*}(U)
$$

given by

$$
\bar{\psi} \longmapsto \bar{\Theta}_{U, \bar{\psi}} \equiv\left(\left(\bar{\Theta}_{U, \bar{\psi}}\right)_{V}\right)_{U \supseteq V, \text { open }}
$$

and such that for any $r \in \mathcal{E}(V)$

$$
\left(\bar{\Theta}_{U, \bar{\psi}}\right)_{V}\left(r+\mathcal{E}_{0}(V)\right):=\bar{\Theta}_{V}\left(r+\mathcal{E}_{0}(V),\left.\bar{\psi}\right|_{V}\right)=\bar{\psi}_{V}(r)=\psi_{V}\left(r+\mathcal{E}_{0}(V)\right)
$$

is the image. Thus the image of $\bar{\psi}$ is $\psi$, hence the map $\mathcal{E}_{0}^{\perp}(U) \longrightarrow\left(\mathcal{E} / \mathcal{E}_{0}\right)^{*}(U)$, derived from (6), is onto, and therefore an $\mathcal{A}(U)$-isomorphism. Since $\mathcal{E} / \mathcal{E}_{0}$ is free by Corollary 1 , so are $\left(\mathcal{E} / \mathcal{E}_{0}\right)^{*}$ and $\mathcal{E}_{0}^{\perp}$ free.

Now, let $\mathcal{F}_{0}$ be a free sub- $\mathcal{A}$-module of $\mathcal{E}^{*} \cong \mathcal{E}$ (cf. Mallios [7, p.298, (5.2)]); on considering $\mathcal{F}_{0}$ as a free sub- $\mathcal{A}$-module of $\mathcal{E}$, according to all that precedes above $\mathcal{F}_{0}^{\perp}$ is free in $\mathcal{E}^{*} \cong \mathcal{E}$, and so the proof is finished. 
Now, if $(\mathcal{E}, \mathcal{F} ; \phi)$ is an orthogonally convenient pairing, $\mathcal{E}_{0}$ and $\mathcal{F}_{0}$ free sub- $\mathcal{A}$ modules of $\mathcal{E}$ and $\mathcal{F}$, respectively, by $(1), \mathcal{E} / \mathcal{F}_{0}^{\perp}$ and $\mathcal{E} / \mathcal{E}_{0}$ are free $\mathcal{A}$-modules. Since the maps in Theorem 2 are $\mathcal{A}$-isomorphisms into,

$$
\operatorname{rank}\left(\mathcal{E} / \mathcal{F}_{0}^{\perp}\right) \leq \operatorname{rank} \mathcal{F}_{0}^{*}=\operatorname{rank} \mathcal{F}_{0}
$$

and

$$
\operatorname{rank} \mathcal{E}_{0}^{\perp} \leq \operatorname{rank}\left(\mathcal{E} / \mathcal{E}_{0}\right)^{*}=\operatorname{rank}\left(\mathcal{E} / \mathcal{E}_{0}\right)
$$

Inequalities (8) and (9) can also be written in the form

$$
\operatorname{corank} \mathcal{F}_{0}^{\perp} \leq \operatorname{rank} \mathcal{F}_{0}
$$

and

$$
\operatorname{rank} \mathcal{E}_{0}^{\perp} \leq \operatorname{corank} \mathcal{E}_{0}
$$

If we put $\mathcal{E}_{0}=\mathcal{F}_{0}^{\perp}$ in the last inequality and combine it with the first one, we get

$$
\operatorname{rank} \mathcal{F}_{0}^{\perp \perp} \leq \operatorname{corank} \mathcal{F}_{0}^{\perp} \leq \operatorname{rank} \mathcal{F}_{0}
$$

But $\mathcal{F}_{0}$ is a free sub- $\mathcal{A}$-module of $\mathcal{F}_{0}^{\perp \perp}$, so that rank $\mathcal{F}_{0} \leq$ rank $\mathcal{F}_{0}^{\perp \perp}$, and (10) becomes

$$
\operatorname{rank} \mathcal{F}_{0}^{\perp \perp}=\operatorname{corank} \mathcal{F}_{0}^{\perp}=\operatorname{rank} \mathcal{F}_{0}
$$

Let us consider the formula (11) in the case where rank $\mathcal{F}_{0}$ is finite. We clearly have $\mathcal{F}_{0}^{\perp \perp}=\mathcal{F}_{0}$ within an $\mathcal{A}$-isomorphism. The $\mathcal{A}$-module $\mathcal{F}_{0}$ is said to be orthogonally reflexive. In the $\mathcal{A}$-morphism (5), both free $\mathcal{A}$-modules have the same finite rank, the $\mathcal{A}$-isomorphism into is, therefore, onto and thus

$$
\mathcal{E} / \mathcal{F}_{0}^{\perp}=\mathcal{F}_{0}^{*}
$$

within an $\mathcal{A}$-isomorphism. Hence, $\mathcal{E} / \mathcal{F}_{0}^{\perp}$ may be regarded naturally as the dual $\mathcal{A}$ module of $\mathcal{F}_{0}$. For the $\mathcal{A}$-morphism (6), put $\mathcal{E}_{0}=\mathcal{F}_{0}^{\perp}$; thus (6) becomes an $\mathcal{A}$-isomorphism

$$
\mathcal{F}_{0}^{\perp \perp} \cong\left(\mathcal{E} / \mathcal{F}_{0}^{\perp}\right)^{*}
$$

Putting $\mathcal{F}_{0}=\mathcal{F}$ in $(11)$, we obtain 
Now, assume in our orthogonally convenient pairing $(\mathcal{E}, \mathcal{F} ; \phi)$ that the right kernel $\mathcal{E}^{\perp}$ is not 0 . Let $\Psi \in \mathrm{Hom}_{\mathcal{A}}\left(\mathcal{E} \oplus\left(\mathcal{F} / \mathcal{E}^{\perp}\right), \mathcal{A}\right)$ such that

$$
\Psi_{U}\left(s, t+\mathcal{E}^{\perp}(U)\right):=\phi_{U}(s, t),
$$

where $U$ is an open subset of $X, t+\mathcal{E}^{\perp}(U) \in\left(\mathcal{F} / \mathcal{E}^{\perp}\right)(U) \cong \mathcal{F}(U) / \mathcal{E}^{\perp}(U)$, (cf. (4)) and $s \in \mathcal{E}(U)$.

The element $t+\mathcal{E}^{\perp}(U)$ lies in the right kernel $\mathcal{E}^{\perp}(U) \cong \mathcal{E}(U)^{\perp}$ if $\phi_{U}(s, t)=0$ for all $s \in \mathcal{E}(U)$. But this means $t \in \mathcal{E}^{\perp}(U)$, so that $t+\mathcal{E}^{\perp}(U)=\mathcal{E}^{\perp}(U)$. It follows that the right kernel of the pairing $\left(\mathcal{E}, \mathcal{F} / \mathcal{E}^{\perp} ; \Psi\right)$ is 0 . The left kernel is obviously the old $\mathcal{F}^{\perp}$. Applying (12), we have

$$
\operatorname{rank}\left(\mathcal{E} / \mathcal{F}^{\perp}\right)=\operatorname{rank}\left(\mathcal{F} / \mathcal{E}^{\perp}\right)
$$

Suppose now that both kernels $\mathcal{E}^{\perp}$ and $\mathcal{F}^{\perp}$ are zero, and that rank $\mathcal{F}$ is finite. (13) shows that $\operatorname{rank} \mathcal{E}$ is also finite and $\operatorname{rank} \mathcal{E}=\operatorname{rank} \mathcal{F}$. So whenever $\mathcal{E}^{\perp}=0=\mathcal{F}^{\perp}$, by [14, Theorem 2.3], we see that each of the free $\mathcal{A}$-modules $\mathcal{F}$ and $\mathcal{E}$ is naturally the dual of the other.

Now, still under the condition $\mathcal{E}^{\perp}=0=\mathcal{F}^{\perp}$ for the orthogonally convenient pairing $(\mathcal{E}, \mathcal{F} ; \phi)$, let us look at the correspondence $\mathcal{F}_{0} \longmapsto \mathcal{F}_{0}^{\perp}$ of a free sub- $\mathcal{A}$-module $\mathcal{F}_{0}$ of $\mathcal{F}$ and the free sub- $\mathcal{A}$-module $\mathcal{F}_{0}^{\perp}$ of $\mathcal{E}$. Any free sub- $\mathcal{A}$-module $\mathcal{E}_{0}$ of $\mathcal{E}$ is obtainable from an $\mathcal{F}_{0}$; indeed we merely have to put $\mathcal{F}_{0}=\mathcal{E}_{0}^{\perp}$. And if $\mathcal{F}_{0} \not \mathcal{F}_{1}$, then $\mathcal{F}_{0}^{\perp} \not \mathcal{F}_{1}^{\perp}$. The correspondence $\mathcal{F}_{0} \longleftrightarrow \mathcal{F}_{0}^{\perp}$, where $\mathcal{F}_{0}$ is any free sub- $\mathcal{A}$-module of $\mathcal{F}$, is one-to-one, and also if $\mathcal{F}_{0} \subseteq \mathcal{F}_{1}$ then $\mathcal{F}_{0}^{\perp} \supseteq \mathcal{F}_{1}^{\perp}$.

Let us collect all our results.

Theorem 4 Let $(\mathcal{E}, \mathcal{F} ; \phi)$ be an orthogonally convenient pairing. Then,

(a) $\operatorname{rank}\left(\mathcal{F} / \mathcal{E}^{\perp}\right)=\operatorname{rank}\left(\mathcal{E} / \mathcal{F}^{\perp}\right)$; in particular if one of the free $\mathcal{A}$-modules $\mathcal{F} / \mathcal{E}^{\perp}$ and $\mathcal{E} / \mathcal{F}^{\perp}$ has finite rank, so has the other one, and the ranks are equal.

(b) If the right kernel $\mathcal{E}^{\perp}$ is zero, and $\mathcal{F}_{0} \subseteq \mathcal{F}$ is a free sub- $\mathcal{A}$-module, then

$$
\operatorname{rank} \mathcal{F}_{0}=\operatorname{corank} \mathcal{F}_{0}^{\perp}=\operatorname{rank} \mathcal{F}_{0}^{\perp \perp} .
$$

If rank $\mathcal{F}_{0}$ is finite, then $\mathcal{F}_{0}^{\perp \perp}=\mathcal{F}_{0}$ and $\mathcal{E} / \mathcal{F}_{0}^{\perp}=\mathcal{F}_{0}^{*}$ within an $\mathcal{A}$-isomorphism, i.e. each of the free $\mathcal{A}$-modules $\mathcal{F}_{0}$ and $\mathcal{E} / \mathcal{F}_{0}^{\perp}$ is naturally the dual of the other.

(c) If both kernels are zero, and rank $\mathcal{F}$ is finite, then $\mathcal{F}=\mathcal{E}$ within an $\mathcal{A}$-isomorphism. The correspondence $\mathcal{F}_{0} \longmapsto \mathcal{F}_{0}^{\perp}$ is a bijection between the free sub- $\mathcal{A}$ modules of $\mathcal{F}$ and the free sub- $\mathcal{A}$-modules of $\mathcal{E}$, and it reverses any inclusion relation.

As a corollary of the preceding theorem, we have

Theorem 5 Let $\left(\mathcal{E}, \mathcal{E}^{*} ; \phi\right)$ be the canonical pairing between (free $\mathcal{A}$-modules) $\mathcal{E}$ and $\mathcal{E}^{*}$, and let $\mathcal{E}_{0}$ be a free $\mathcal{A}$-module of $\mathcal{E}$. Then $\mathcal{E}_{0}^{\perp \perp}=\mathcal{E}_{0}$ and $\mathcal{E}_{0}^{\perp}=\left(\mathcal{E} / \mathcal{E}_{0}\right)^{*}$ within an $\mathcal{A}$-isomorphism, and rank $\mathcal{E}_{0}^{\perp}=$ corank $\mathcal{E}_{0}$. The correspondence $\mathcal{F}_{0} \longmapsto \mathcal{F}_{0}^{\perp}$ is a bijection between free sub-A-modules $\mathcal{F}_{0} \subseteq \mathcal{E}^{*}$ of finite rank and all the free sub- $\mathcal{A}$-modules of $\mathcal{E}$ with finite corank. 
Before stating the theorem, let us recall the result, see [18], that given an $\mathcal{A}$-module $\mathcal{E}$, equipped with an orthosymmetric $\mathcal{A}$-bilinear form $\phi: \mathcal{E} \oplus \mathcal{E} \longrightarrow \mathcal{A}$, then for every open subset $U$ of $X, \phi_{U}$ is either symmetric or skew-symmetric. When $\phi_{U}$ is symmetric, the geometry on the $\mathcal{A}(U)$-module $\mathcal{E}(U)$ is called orthogonal; on the other hand, if $\phi_{U}$ is skew-symmetric, the geometry is called symplectic. No other case can occur if $\phi$ must be orthosymmetric.

Lemmas 2 and 3, below, are needed for the proof of the Witt's theorem. Proofs of Lemmas 2 and 3 are found in [15].

Lemma 2 Let $(\mathcal{E}, \phi)$ be a free $\mathcal{A}$-module of rank 2, endowed with a non-degenerate symmetric or antisymmetric $\mathcal{A}$-bilinear form $\phi$. For an open subset $U \subseteq X$, the nonisotropic $\mathcal{A}(U)$-plane $\mathcal{E}(U)$ is hyperbolic if it contains a nowhere-zero isotropic section $r$.

Lemma 3 Let $(\mathcal{E}, \phi)$ be a non-isotropic convenient $\mathcal{A}$-module, where $\mathcal{A}$ is a PID algebra sheaf, and $F$ any free sub- $\mathcal{A}(U)$-module of $\mathcal{E}(U)$. Moreover, let sections $s_{1}, s_{2}, \ldots, s_{k} \in F$ form a basis of rad $F$ and $G$ a free sub- $\mathcal{A}(U)$-module of $\mathcal{E}(U)$ such that $F=\operatorname{rad} F \perp G$. Then, there are isotropic sections $t_{1}, t_{2}, \ldots, t_{k} \in \mathcal{E}(U)$ such that the planes $P_{i}:=\left[s_{i}, t_{i}\right]$ are hyperbolic, pairwise orthogonal and also orthogonal to $\mathcal{G}(U)$. The $\mathcal{A}(U)$-module

$$
P_{1} \perp P_{2} \perp \ldots \perp P_{k} \perp G
$$

contains $F$.

On the basis of Lemma 3, above, we introduce the following notion.

Definition 5 Let $(\mathcal{E}, \phi)$ be a non-isotropic convenient $\mathcal{A}$-module, and $\mathcal{F}$ a free sub$\mathcal{A}$-module of $\mathcal{E}$ of rank $k$. The free sub- $\mathcal{A}$-module $\mathcal{F}$ is called pre-hyperbolic if there are pairwise orthogonal hyperbolic $\mathcal{A}$-planes

$$
\mathcal{H}_{1}, \mathcal{H}_{2}, \ldots, \mathcal{H}_{l} \subseteq \mathcal{E}
$$

such that if $\mathcal{F}=\operatorname{rad} \mathcal{F} \perp \mathcal{G}$ with rad $\mathcal{F} \cong \mathcal{A}^{l}$ and $\mathcal{G} \cong \mathcal{A}^{k-l}$, then

$$
\mathcal{H}_{1} \perp \mathcal{H}_{2} \perp \ldots \perp \mathcal{H}_{l} \perp \mathcal{G}
$$

is non-isotropic and contains $\mathcal{F}$.

Theorem 6 (Witt's Theorem) Let $(\mathcal{E}, \phi)$ be a non-isotropic convenient $\mathcal{A}$-module, with the $\mathcal{A}$-bilinear form $\phi$ symmetric or antisymmetric. Let $\mathcal{F}$ and $\mathcal{F}^{\prime}$ be pre-hyperbolic free sub- $\mathcal{A}$-modules of $\mathcal{E}$, and let $\sigma \in \operatorname{Hom}_{\mathcal{A}}\left(\mathcal{F}, \mathcal{F}^{\prime}\right)$ be an isomtery. Then, $\sigma$ extends to an isometry of $\mathcal{E}$ onto itself. 
Proof For every open set $U \subseteq X, \sigma_{U}: \mathcal{F}(U) \longrightarrow \mathcal{F}^{\prime}(U)$ is an $\mathcal{A}(U)$-isometry. Suppose that $\sigma_{U}$ extends to an isometry $\widehat{\sigma}_{U}: \mathcal{E}(U) \longrightarrow \mathcal{E}(U)$ such that $\rho_{V}^{U} \circ \widehat{\sigma}_{U}=\widehat{\sigma}_{V} \circ \rho_{V}^{U}$, where the $\rho_{V}^{U}$ are restriction maps for $\mathcal{E}$, then the $\mathcal{A}$-morphism $\beta: \mathcal{E} \longrightarrow \mathcal{E}$ such that $\beta_{U}:=\widehat{\sigma}_{U}$ is an $\mathcal{A}$-isometry of $\mathcal{E}$ onto itself and extends $\sigma$.

We shall see that the proof reduces to the case when $\mathcal{F}$ is non-isotropic. For, suppose $\mathcal{F}$ is isotropic, and $\mathcal{F}=\operatorname{rad} \mathcal{F} \perp \mathcal{G}$, where $\operatorname{rad} \mathcal{F} \cong \mathcal{A}^{l}$ and $\mathcal{G} \cong \mathcal{A}^{k-l}$. Since $\sigma \in \operatorname{Hom}_{\mathcal{A}}\left(\mathcal{F}, \mathcal{F}^{\prime}\right)$ is an isometry, for every open $U \subseteq X, \mathcal{F}^{\prime}(U)=\sigma_{U}(\mathcal{F}(U))=$ $\sigma_{U}\left((\operatorname{rad} \mathcal{F})(U) \perp \sigma_{U}(\mathcal{G}(U))=\sigma_{U}(\operatorname{rad} \mathcal{F}(U)) \perp \sigma_{U}(\mathcal{G}(U))\right.$. Clearly, $\operatorname{rad} \mathcal{F}^{\prime}(U)=$ $\sigma_{U}(\operatorname{rad} \mathcal{F}(U))$. Now, based on the fact that both $\mathcal{F}$ and $\mathcal{F}^{\prime}$ are pre-hyperbolic, we can enlarge them to orthogonal sums

$$
\mathcal{H}_{1} \perp \cdots \perp \mathcal{H}_{l} \perp \mathcal{G} \quad \text { and } \quad \mathcal{H}_{1}^{\prime} \perp \cdots \perp \mathcal{H}_{l}^{\prime} \perp \sigma(\mathcal{G})
$$

where, for every open subset $U \subseteq X$, and $1 \leq i \leq l$,

$$
\mathcal{H}_{i}(U)=\left[e_{i, U}, f_{i, U}\right]
$$

in keeping with the notations of Definition 5, and if $\sigma_{U}\left(e_{i, U}\right)=e_{i, U}^{\prime}$, we can find $f_{i, U}^{\prime} \in \mathcal{F}^{\prime}(U)$ such that,

$$
\mathcal{H}_{i}^{\prime}(U)=\left[e_{i, U}^{\prime}, f_{i, U}^{\prime}\right]
$$

is an hyperbolic $\mathcal{A}(U)$-plane, orthogonal to $\mathcal{G}^{\prime}(U):=\sigma_{U}(\mathcal{G}(U)) \cong \mathcal{A}^{k-l}(U) \cong$ $\mathcal{A}(U)^{k-l}$. We extend every $\sigma_{U}$ to an $\mathcal{A}$-isometry

$$
\bar{\sigma}_{U}: \mathcal{H}_{1}(U) \perp \cdots \perp \mathcal{H}_{l}(U) \perp \mathcal{G}(U) \longrightarrow \mathcal{H}_{1}^{\prime}(U) \perp \cdots \perp \mathcal{H}_{l}^{\prime}(U) \perp \mathcal{G}^{\prime}(U)
$$

by requiring that $\bar{\sigma}_{U}\left(f_{i, U}\right)=f_{i, U}^{\prime}$. The $\mathcal{A}$-morphism $\bar{\sigma} \equiv\left(\bar{\sigma}_{U}\right)$ thus obtained is an $\mathcal{A}$-isometry

$$
\mathcal{H}_{1} \perp \cdots \perp \mathcal{H}_{l} \perp \mathcal{G} \longrightarrow \mathcal{H}_{1}^{\prime} \perp \cdots \perp \mathcal{H}_{l}^{\prime} \perp \mathcal{G}^{\prime}
$$

But $\mathcal{H}_{1} \perp \cdots \perp \mathcal{H}_{l} \perp \mathcal{G}$ and $\mathcal{H}_{1}^{\prime} \perp \cdots \perp \mathcal{H}_{l}^{\prime} \perp \mathcal{G}^{\prime}$ are non-isotropic, so the proof is reduced to the anticipated case, viz. the case when $\mathcal{F}$ is non-isotropic.

(A) Symplectic geometry By Theorem 1 , since $\mathcal{F}$ and $\mathcal{F}^{\prime}$ are non-isotropic

$$
\mathcal{E}=\mathcal{F} \perp \mathcal{F}^{\perp} \text { and } \mathcal{E}=\mathcal{F}^{\prime} \perp \mathcal{F}^{\prime}
$$

We need only show that $\mathcal{F}^{\perp}$ and $\mathcal{F}^{\prime} \perp$ are $\mathcal{A}$-isometric; in fact $\mathcal{F}^{\perp}$ and $\mathcal{F}^{\prime}$ are free non-isotropic sub- $\mathcal{A}$-modules of $\mathcal{E}$ of the same rank. Moreover, by Theorem 1 , since restrictions $\left.\phi\right|_{\mathcal{F}^{\perp}}$ and $\left.\phi\right|_{\mathcal{F}^{\prime} \perp}$ (of $\phi$ to $\mathcal{F}^{\perp}$ and $\mathcal{F}^{{ }^{\perp}}$, respectively) are non-degenerate, $\mathcal{F}^{\perp}$ and $\mathcal{F}^{\prime}$ are direct orthogonal sums of hyperbolic $\mathcal{A}$-planes. Based on the latter observations, $\mathcal{F}^{\perp}$ and $\mathcal{F}^{\prime} \perp$ are $\mathcal{A}$-isometric. 
(B) Orthogonal geometry We will proceed stepwise. Case (1) Suppose that $\mathcal{F}=$ $\mathcal{F}^{\prime}$, i.e. $\sigma$ is an $\mathcal{A}$-isometry of $\mathcal{F}$ onto itself. We extend $\sigma$ by keeping, for every open $U \subseteq X$, every section in $\mathcal{F}^{\perp}(U)$ fixed. Case (2) Assume rank $\mathcal{F}=$ rank $\mathcal{F}^{\prime}=1$, i.e. $\mathcal{F} \cong \mathcal{F}^{\prime} \cong \mathcal{A}$, and $\mathcal{F} \neq \mathcal{F}^{\prime}$. Thus, for some open subset $U \subseteq X, \mathcal{F}(U) \neq \mathcal{F}^{\prime}(U)$. Say $\mathcal{F}(U)=\left[e_{U}\right]$ and $\mathcal{F}^{\prime}(U)=\left[e_{U}^{\prime}\right]$ for every open $U \subseteq X . \mathcal{F}$ and $\mathcal{F}^{\prime}$ being $\mathcal{A}$-modules of rank 1, it is clear that if $U$ and $V$ are open sets in $X$ such that $V \subseteq U$, then $e_{V}=\left.e_{U}\right|_{V}$ and $e_{V}^{\prime}=\left.e_{U}^{\prime}\right|_{V}$. Next, since $\mathcal{F}=\mathcal{F}^{\prime}$ within an $\mathcal{A}$-isometry, and $\mathcal{F}$ and $\mathcal{F}^{\prime}$ are non-isotropic, we have that $\phi_{U}\left(e_{U}, e_{U}\right)=\phi_{U}\left(e_{U}^{\prime}, e_{U}^{\prime}\right) \neq 0$, for every open $U \subseteq X$. Furthermore, the correspondence

$$
U \longmapsto \mathcal{J}(U):=\left[e_{U}, e_{U}^{\prime}\right]
$$

along with the obvious restriction maps (in fact, the restriction maps $\delta_{V}^{U}$ are given by the prescription $\delta_{V}^{U}\left(a e_{U}+b e_{U}^{\prime}\right)=\lambda_{V}^{U}(a) \rho_{V}^{U}\left(e_{U}\right)+\lambda_{V}^{U}(b) \rho_{V}^{U}\left(e_{U}^{\prime}\right)$ for every $a, b \in \mathcal{A}(U)$ ) yields a presheaf of $\mathcal{A}(U)$-modules. For an open subset $U \subseteq X$ such that $\mathcal{F}(U)=\mathcal{F}^{\prime}(U)$, it follows that $\mathcal{J}(U)$ is of rank 1 and non-isotropic. In this case, since $\mathcal{E}(U)=\mathcal{J}(U) \perp \mathcal{J}(U)^{\perp}, \sigma_{U}$ is extended by keeping the sections in $\mathcal{J}(U)^{\perp}$ fixed. On the other hand, for an open $U$ such that $\mathcal{J}(U) \equiv\left[e_{U}, e_{U}^{\prime}\right]$ has rank 2 , we distinguish two situations.

If $\mathcal{J}(U)$ is non-isotropic, the map, which sends $e_{U}$ to $e_{U}^{\prime}$ and $e_{U}^{\prime}$ to $e_{U}$, is an $\mathcal{A}(U)$ isometry. Then, we apply Case (1) to get an $\mathcal{A}(U)$-isometry $\beta_{U}$ of $\mathcal{E}(U)$ onto itself. Clearly for each open $U \subseteq X$,

$$
\beta_{V} \circ \rho_{V}^{U}=\rho_{V}^{U} \circ \beta_{U} .
$$

Hence, $\beta \equiv\left(\beta_{U}\right)$ is an $\mathcal{A}$-isometry extending $\sigma$.

Next, if $\mathcal{J}(U)$ is isotropic, then $\operatorname{rad} \mathcal{J}(U)$ has rank 1. Let $s_{U}$ be a generator of rad $\mathcal{J}(U)$. There exist nowhere-zero sections $a, b \in \mathcal{A}(U)$ such that $e_{U}^{\prime}=a e_{U}+b s_{U}$. Then, $\phi_{U}\left(e_{U}^{\prime}, e_{U}^{\prime}\right)=a^{2} \phi_{U}\left(e_{U}, e_{U}\right)$ and because $\phi_{U}\left(e_{U}^{\prime}, e_{U}^{\prime}\right)$ and $\phi_{U}\left(e_{U}, e_{U}\right)$ are nowhere-zero sections of $\mathcal{A}$ on $U$, it follows that $a= \pm 1$. Assume that $a=1$, and let us replace $b s_{U}$ by $s_{U}$, then

$$
s_{U}=e_{U}^{\prime}-e_{U}
$$

Let $t_{U}=e_{U}+e_{U}^{\prime}$. It is obvious that $\left[s_{U}, t_{U}\right]=\left[s_{U}\right] \oplus\left[t_{U}\right]$ and $\operatorname{rad}\left(\left[s_{U}, t_{U}\right]\right)=\left[s_{U}\right]$; by Lemma 3, we can find a section $z_{U} \in \mathcal{E}(U)$ such that

$$
\phi_{U}\left(z_{U}, t_{U}\right)=0, \quad \phi_{U}\left(z_{U}, z_{U}\right)=0 \text { and } \phi_{U}\left(s_{U}, z_{U}\right)=1 .
$$

The $\mathcal{A}(U)$-module $\left[t_{U}\right] \perp\left[s_{U}, z_{U}\right]$ is non-isotropic, being an orthogonal sum of $\left[t_{U}\right]$ and the hyperbolic plane $\left[s_{U}, z_{U}\right]$. There exists an $\mathcal{A}(U)$-isometry such that 
But $e_{U}=\frac{1}{2}\left(t_{U}-s_{U}\right)$ is mapped on $e_{U}^{\prime}=\frac{1}{2}\left(t_{U}+s_{U}\right)$ by this isometry. Resorting to Case (1), we obtain an $\mathcal{A}(U)$-isometry $\mathcal{E}(U) \longrightarrow \mathcal{E}(U)$ which extends $\sigma_{U}$. This part of the proof is therefore finished.

Case (3) We finish the proof by induction. Let $\mathcal{F}=\mathcal{F}_{1} \perp \mathcal{F}_{2}$, where $\mathcal{F}_{1}$ and $\mathcal{F}_{2}$ are free sub- $\mathcal{A}$-modules of $\mathcal{E}$ of rank greater than or equal to 1 . Then, $\mathcal{F}_{2} \subseteq \mathcal{F}_{1}^{\perp}$, consequently by applying Lemma 1 , we have that $\mathcal{F}_{2} \cap \mathcal{F}_{2}^{\perp} \subseteq \mathcal{F}_{1}^{\perp} \cap \mathcal{F}_{2}^{\perp}=\mathcal{F}^{\perp}$. It easily follows that

$$
\mathcal{F} \cap \mathcal{F}_{2} \cap \mathcal{F}_{2}^{\perp}=\mathcal{F}_{2} \cap \mathcal{F}_{2}^{\perp} \subseteq \mathcal{F} \cap \mathcal{F}^{\perp}=0
$$

i.e. $\mathcal{F}_{2}$ is non-isotropic. One applies a similar argument to show that $\mathcal{F}_{1}$ is also nonisotropic. Now, let us fix an open subset $U \subseteq X$. We have $\mathcal{F}(U)=\mathcal{F}_{1}(U) \perp \mathcal{F}_{2}(U)$ and

$$
\sigma_{U}(\mathcal{F}(U))=\sigma_{U}\left(\mathcal{F}_{1}(U)\right) \perp \sigma_{U}\left(\mathcal{F}_{2}(U)\right)
$$

Let $\sigma_{1, U}=\sigma_{U} \mid \mathcal{F}_{1}(U)$ be the restriction of $\sigma_{U}$ to $\mathcal{F}_{1}(U)$. By induction, we can extend $\sigma_{1, U}$ to an $\mathcal{A}(U)$-isometry

$$
\bar{\sigma}_{1, U}: \mathcal{E}(U) \longrightarrow \mathcal{E}(U)
$$

Then, $\bar{\sigma}_{1, U}\left(\mathcal{F}_{1}^{\perp}(U)\right)=\left(\sigma_{1, U}\left(\mathcal{F}_{1}(U)\right)\right)^{\perp}$. Indeed, let $r \in \mathcal{F}_{1}^{\perp}(U)$ and $s \in \mathcal{F}_{1}(U)$. Then

$$
\phi_{U}\left(\bar{\sigma}_{1, U}(r), \sigma_{1, U}(s)\right)=\phi_{U}\left(\bar{\sigma}_{1, U}(r), \bar{\sigma}_{1, U}(s)\right)=\phi_{U}(r, s)=0 .
$$

Thus, $\bar{\sigma}_{1, U}(r) \in\left(\sigma_{1, U}\left(\mathcal{F}_{1}(U)\right)\right)^{\perp}$ and hence

$$
\bar{\sigma}_{1, U}\left(\mathcal{F}_{1}^{\perp}(U)\right) \subseteq\left(\sigma_{1, U}\left(\mathcal{F}_{1}(U)\right)\right)^{\perp}
$$

Conversely, let $r \in\left(\sigma_{1, U}\left(\mathcal{F}_{1}(U)\right)\right)^{\perp}$. Then $\phi_{U}\left(r, \sigma_{1, U}(s)\right)=0, s \in \mathcal{F}_{1}(U)$. Since $\bar{\sigma}_{1, U}: \mathcal{E}(U) \longrightarrow \mathcal{E}(U)$ is an isometry and $\sigma_{1, U}=\bar{\sigma}_{1, U} \mid \mathcal{F}_{1(U)}$, one has, given that $r=\bar{\sigma}_{1, U}(t)$ for some $t \in \mathcal{E}(U)$,

$$
\phi_{U}\left(\bar{\sigma}_{1, U}(t), \bar{\sigma}_{1, U}(s)\right)=0
$$

which, in turn, implies

$$
\phi_{U}(t, s)=0
$$

Consequently, $t \in\left(\mathcal{F}_{1}(U)\right)^{\perp}=\mathcal{F}_{1}^{\perp}(U)$. But $r:=\bar{\sigma}_{1, U}(t) \in \bar{\sigma}_{1, U}\left(\mathcal{F}_{1}^{\perp}(U)\right)$, therefore

$$
\left(\sigma_{1, U}\left(\mathcal{F}_{1}(U)\right)\right)^{\perp} \subseteq \bar{\sigma}_{1, U}\left(\mathcal{F}_{1}^{\perp}(U)\right)
$$


Since $\sigma_{U}\left(\mathcal{F}_{2}(U)\right)$ is orthogonal to $\sigma_{U}\left(\mathcal{F}_{1}(U)\right):=\sigma_{1, U}\left(\mathcal{F}_{1}(U)\right)$, it follows that $\sigma_{U}\left(\mathcal{F}_{2}(U)\right) \subseteq \bar{\sigma}_{1, U}\left(\mathcal{F}_{1}^{\perp}(U)\right)$. Let $\sigma_{2, U}=\sigma_{U} \mid \mathcal{F}_{2}(U)$. Then, the $\mathcal{A}(U)$-isometry

$$
\sigma_{2, U}: \mathcal{F}_{2}(U) \longrightarrow \sigma_{2, U}\left(\mathcal{F}_{2}(U)\right):=\sigma_{U}\left(\mathcal{F}_{2}(U)\right)
$$

extends by induction to an $\mathcal{A}(U)$-isometry

$$
\bar{\sigma}_{2, U}: \mathcal{F}_{1}^{\perp}(U) \longrightarrow \bar{\sigma}_{1, U}\left(\mathcal{F}_{1}^{\perp}(U)\right)
$$

The pair $\left(\sigma_{1, U}, \bar{\sigma}_{2, U}\right)$ applies isometrically $\mathcal{F}_{1}(U) \perp \mathcal{F}_{1}^{\perp}(U)=\mathcal{E}(U)$ onto itself, as desired. Since $\mathcal{F}_{1}$ and $\mathcal{F}_{1}^{\perp}$ are sub- $\mathcal{A}$-modules of $\mathcal{E}$ and each diagram

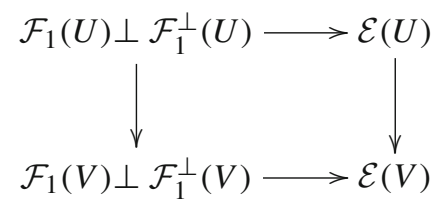

where $U$ and $V$ are open subsets of $X$ such that $V \subseteq U$, is commutative, $\left(\sigma_{1}, \bar{\sigma}_{2}\right) \equiv$ $\left(\sigma_{1, U}, \bar{\sigma}_{2, U}\right)$ is an $\mathcal{A}$-isometry of $\mathcal{E}$ onto $\mathcal{E}$, and the proof is finished.

\section{Witt's theorem second version}

Unlike Theorem 6 , in which the $\mathcal{A}$-bilinear morphism $\phi$ may be symmetric or antisymmetric, the $\mathcal{A}$-bilinear morphism of Theorem 7, below, is assumed to be symmetric case and free sub- $\mathcal{A}$-modules $\mathcal{F}_{1}$ and $\mathcal{F}_{2}$ of the convenient $\mathcal{A}$-module $\mathcal{E}$ are disjoint and need not be pre-hyperbolic.

Theorem 7 Let $\phi \equiv\left(\phi_{U}\right)$ be a symmetric $\mathcal{A}$-bilinear form on a non-isotropic convenient $\mathcal{A}$-module $\mathcal{E}$ of rank $m \geq 2$. Let $\mathcal{F}_{1}$ and $\mathcal{F}_{2}$ be strongly non-isotropic free sub-A-modules of $\mathcal{E}$ such that $\mathcal{F}_{1} \cap \mathcal{F}_{2}=0$, and let $\sigma: \mathcal{F}_{1} \longrightarrow \mathcal{F}_{2}$ be an $\mathcal{A}$-isometry. Then $\mathcal{F}_{1}^{\perp}$ and $\mathcal{F}_{2}^{\perp}$ are isometric.

Proof We prove the theorem by induction on $n=\operatorname{rank}\left(\mathcal{F}_{1}\right)=\operatorname{rank}\left(\mathcal{F}_{2}\right)$. First, let $\Gamma(\mathcal{E}) \equiv\left(\mathcal{E}(U), \rho_{V}^{U}\right)$ and $\Gamma(\mathcal{A}) \equiv\left(\mathcal{A}(U), \lambda_{V}^{U}\right)$ denote, as usual, the (complete)presheaf of sections of $\mathcal{E}$ and $\mathcal{A}$, respectively.

Let $n=1, m=2, r_{1} \equiv r_{1, X}$ generate $\mathcal{F}_{1}(X)$, and $r_{2} \equiv r_{2, X}:=\sigma_{X}\left(r_{1}\right)$ generate $\mathcal{F}_{2}(X)$; since $\mathcal{F}_{1}$ and $\mathcal{F}_{2}$ are non-isotropic and isometric,

$$
a:=\phi_{X}\left(r_{1}, r_{1}\right)=\phi_{X}\left(r_{2}, r_{2}\right) \neq 0
$$

in the sense that $\left.\lambda_{U}^{X}(a) \equiv a\right|_{U} \neq 0$ for any open subset $U$ of $X$. On using Theorem 1, $\mathcal{E}=\mathcal{F}_{i} \perp \mathcal{F}_{i}^{\perp}$; so $\mathcal{E}(X)$ has bases $B_{i, X}=\left\{r_{i}, r_{i}^{\perp}\right\}$ where $r_{i}^{\perp}$ spans $\mathcal{F}_{i}^{\perp}(X)$. Suppose that $\phi_{X}\left(r_{1}, r_{2}\right)=\phi_{X}\left(r_{1}, r_{1}\right)=\phi_{X}\left(r_{2}, r_{2}\right)$. Clearly, $r_{1}-r_{2} \in \mathcal{F}_{1}^{\perp}(X)$ and $\mathcal{F}_{1}^{\perp}(X)=$ $\mathcal{F}_{2}^{\perp}(X)$. Since $\mathcal{E}$ is convenient, we have $\mathcal{F}_{1}(X)=\mathcal{F}_{1}^{\perp \perp}(X)=\mathcal{F}_{2}^{\perp \perp}(X)=\mathcal{F}_{2}(X)$, which is impossible according to our hypothesis. Thus the matrix representing $\phi_{X}$ 
with respect to the basis $\left\{r_{1}, r_{2}\right\}$ is non-singular. By Adkins-Weintraub [1, Theorem 2.21 , p. 3.57], the matrix $\left[\phi_{X}\right]_{B_{i, X}}$ representing $\phi_{X}$ with respect to the basis $B_{i, X}$ is non-singular, and consequently $\mathcal{F}_{i}^{\perp}(X)$ is non-isotropic. Hence,

$$
\left[\phi_{X}\right]_{B_{i, X}}=\operatorname{diag}\left(a, b_{i}\right) \text { for } i=1,2,
$$

with $b_{i}:=\phi_{X}\left(r_{i}^{\perp}, r_{i}^{\perp}\right)$ a nowhere-zero section in $\mathcal{A}(X)$. By Adkins-Weintraub [1, Theorem 2.13, p. 354], there is an invertible matrix $P$ with

$$
\left[\phi_{X}\right]_{B_{2, X}}=P^{t}\left[\phi_{X}\right]_{B_{1, X}} P,
$$

and taking determinants shows that $a b_{2}=c^{2} a b_{1}$ (where $c:=\operatorname{det} P$ is a nowhere-zero section in $\mathcal{A}(X)$ ); so $f \equiv f_{X}: \mathcal{F}_{1}^{\perp}(X) \longrightarrow \mathcal{F}_{2}^{\perp}(X)$ defined by $f_{X}\left(r_{1}^{\perp}\right)=c^{-1} r_{2}^{\perp}$ yields an isometry.

Let $B_{i, U}:=\left\{\left.r_{i}\right|_{U} \equiv r_{i, U}\right\}(i=1,2) ; B_{i, U}$ is a basis of $\mathcal{F}_{i}(U)$. Likewise, let $\left(B_{i, U}^{\perp}\right)=\left\{\left.r_{i}^{\perp}\right|_{U} \equiv r_{i, U}^{\perp}\right\} ;\left(B_{i, U}^{\perp}\right)$ is a basis of $\mathcal{F}_{i}^{\perp}(U)$. Fix an open set $U$ in $X$, the matrices $\left[\phi_{U}\right]_{B_{1, U}}$ and $\left[\phi_{U}\right]_{B_{2, U}}$ relate to each other as follows:

$$
\left[\phi_{U}\right]_{B_{2, U}}=M_{n}\left(\lambda_{U}^{X}\right)\left(P^{t}\right)\left[\phi_{U}\right]_{B_{1, U}} M_{n}\left(\lambda_{U}^{X}\right)(P),
$$

where if $P=\left(p_{i j}\right)_{1 \leq i, j \leq 2}$, then

$$
M_{n}\left(\lambda_{U}^{X}\right)(P)=\left(\lambda_{U}^{X}\left(p_{i j}\right)\right),
$$

cf. Mallios [7, (1.7), p. 281]. Clearly, $\operatorname{det} M_{n}\left(\lambda_{U}^{X}\right)(P)=\left.\lambda_{U}^{X}(c) \equiv c\right|_{U}$; so $f_{U}$ : $\mathcal{F}_{1}^{\perp}(U) \longrightarrow \mathcal{F}_{2}^{\perp}(U)$, defined by setting that $f_{U}\left(r_{1, U}^{\perp}\right)=\left(\left.c\right|_{U}\right)^{-1} r_{2, U}^{\perp}$ gives rise to an isometry between $\mathcal{F}_{1}^{\perp}(U)$ and $\mathcal{F}_{2}^{\perp}(U)$. But

$$
\rho_{V}^{U} \circ f_{U}=f_{V} \circ \rho_{V}^{U}
$$

therefore $f \equiv\left(f_{U}\right): \mathcal{F}_{1}^{\perp} \longrightarrow \mathcal{F}_{2}^{\perp}$ is an $\mathcal{A}$-morphism, from which one derives an isometry between $\mathcal{F}_{1}^{\perp}$ and $\mathcal{F}_{2}^{\perp}$; hence this part of the proof is finished.

Now, we apply induction on $n$. Assume that the theorem is true for

$$
\operatorname{rank}\left(\mathcal{F}_{1}\right)=\operatorname{rank}\left(\mathcal{F}_{2}\right)<n
$$

and let

$$
\operatorname{rank}\left(\mathcal{F}_{1}\right)=\operatorname{rank}\left(\mathcal{F}_{2}\right)=n
$$

First, we claim that there is an $r \in \mathcal{F}_{1}(X)$ with $\phi_{X}(r, r) \neq 0$ for every open $U \subseteq X$. Indeed, let $s \in \mathcal{F}_{1}(X)$. If $\phi_{X}(s, s) \neq 0$, set $r=s$. If $\phi_{X}(s, s)=0$, pick $t \in \mathcal{F}_{1}(X)$ with $\phi_{X}(s, t) \neq 0$. Such a section $t$ exists because $\mathcal{F}_{1}$ is assumed to be non-isotropic. If $\phi_{X}(t, t) \neq 0$, set $r=t$. Otherwise, note that for any $a \in \mathcal{A}(X)$,

$$
\phi_{X}(a s+t, a s+t)=2 a \phi_{X}(s, t) .
$$

Setting $r:=a s+t$, with $a=\left(\phi_{X}(s, t)\right)^{-1}$, we have $\phi_{X}(r, r) \neq 0$. 
On the basis of the preceding argument, let $r_{1} \in \mathcal{F}_{1}(X)$ with $\phi_{X}\left(r_{1}, r_{1}\right) \neq 0$, and let $r_{2}:=\sigma_{X}\left(r_{1}\right) \in \mathcal{F}_{2}(X)$; so

$$
\phi_{X}\left(r_{2}, r_{2}\right)=\phi_{X}\left(r_{1}, r_{1}\right) \neq 0
$$

Let $\mathcal{F}_{11}$ and $\mathcal{F}_{21}$ be sub- $\mathcal{A}$-modules of $\mathcal{E}$ generated by $r_{1}$ and $r_{2}$, respectively. Then

$$
\mathcal{E}=\mathcal{F}_{11} \perp \mathcal{F}_{11}^{\perp}=\mathcal{F}_{21} \perp \mathcal{F}_{21}^{\perp}
$$

or

$$
\mathcal{E}=\mathcal{F}_{11} \perp\left(\mathcal{F}_{11}^{\perp} \cap \mathcal{F}_{1}\right) \perp \mathcal{F}_{1}^{\perp}=\mathcal{F}_{21} \perp\left(\mathcal{F}_{21}^{\perp} \cap \mathcal{F}_{2}\right) \perp \mathcal{F}_{2}^{\perp}
$$

But

$$
\mathcal{F}_{1}=\mathcal{F}_{11} \perp\left(\mathcal{F}_{11}^{\perp} \cap \mathcal{F}_{1}\right)
$$

and

$$
\mathcal{F}_{2}=\mathcal{F}_{21} \perp\left(\mathcal{F}_{21}^{\perp} \cap \mathcal{F}_{2}\right)
$$

since $\sigma: \mathcal{F}_{1} \longrightarrow \mathcal{F}_{2}$ and $\left.\sigma\right|_{\mathcal{F}_{11}}: \mathcal{F}_{11} \longrightarrow \mathcal{F}_{21}$ are $\mathcal{A}$-isometries, it follows that $\mathcal{F}_{11}^{\perp} \cap \mathcal{F}_{1}$ and $\mathcal{F}_{21}^{\perp} \cap \mathcal{F}_{2}$ are isometric to each other. Moreover, it is easy to see that $\mathcal{F}_{11}^{\perp} \cap \mathcal{F}_{1}$ and $\mathcal{F}_{21}^{\perp} \cap \mathcal{F}_{2}$ are non-isotropic and of rank smaller than $n$. (in fact, $r_{1} \notin$ $\mathcal{F}_{11}^{\perp}(X)$ and $r_{2} \notin \mathcal{F}_{21}^{\perp}(X)$.) Next, observe that

$$
\left(\mathcal{F}_{11}^{\perp} \cap \mathcal{F}_{1}\right)^{\perp}=\mathcal{F}_{11} \perp \mathcal{F}_{1}^{\perp}
$$

and

$$
\left(\mathcal{F}_{21}^{\perp} \cap \mathcal{F}_{2}\right)^{\perp}=\mathcal{F}_{21} \perp \mathcal{F}_{2}^{\perp}
$$

So, by applying the inductive hypothesis on both $\mathcal{F}_{11}^{\perp} \cap \mathcal{F}_{1}$ and $\mathcal{F}_{21}^{\perp} \cap \mathcal{F}_{2}$ we note that $\mathcal{F}_{11} \perp \mathcal{F}_{1}^{\perp}$ and $\mathcal{F}_{21} \perp \mathcal{F}_{2}^{\perp}$ are isometric to each other, and consequently $\mathcal{F}_{1}^{\perp}$ and $\mathcal{F}_{2}^{\perp}$ are isometric; the theorem is proved.

\section{References}

1. Adkins, W.A., Weintraub, S.H.: Algebra. An Approach via Module Theory. Springer, New York (1992)

2. Artin, E.: Geometric Algebra. Interscience, New York (1988)

3. Berndt, R.: An Introduction to Symplectic Geometry. American Mathematical Society, Providence, Rhode Island (2001)

4. Crumeyrolle, A.: Orthogonal and Symplectic Clifford Algebras. Spinor Structures. Kluwer, Dordrecht (1990)

5. Deheuvels, R.: Formes Quadratiques et Groupes Classiques. Presses Universitaires de France, Paris (1981)

6. Lang, S.: Algebra. Revised 3rd edn. Springer, New York (2002) 
7. Mallios, A.: Geometry of Vector Sheaves. An Axiomatic Approach to Differential Geometry. Volume I: Vector Sheaves. General Theory. Kluwer, Netherlands (1998)

8. Mallios, A.: Geometry of Vector Sheaves. An Axiomatic Approach to Differential Geometry. Volume II: Geometry. Examples and Applications. Kluwer, Netherlands (1998)

9. Mallios, A. : On an axiomatic treatement of differential geometry via. Vector sheaves. Applications. Math. Jpn. Plaza 48, 93-180 (invited paper) (1998)

10. Mallios, A., Rosinger, E.E.: Space-time foam dense singularities and de rham cohomology. Acta. Appl. Math. 67, 59-89 (2001)

11. Mallios, A.: Quantum gravity and singularities. Note Mat. 25, 57-76 (2005/2006)

12. Mallios, A.: Geometry and physics today. Int. J. Theor. Phys. 45, 1557-1593 (2006)

13. Mallios, A.: $\mathcal{A}$-invariance: an axiomatic approach to quantum relativity. Int. J. Theor. Phys. 47, 19291948 (2008)

14. Mallios, A., Ntumba, P.P.: Pairings of sheaves of $\mathcal{A}$-modules. Quaest. Math. 31, 397-414 (2008)

15. Mallios, A., Ntumba, P.P.: On a sheaf-theoretic version of the Witt's decomposition theorem. A Lagrangian perspective. Rend. Circ. Mat. Palermo 58, 155-168 (2009)

16. Mallios, A., Ntumba, P.P.: Fundamentals for symplectic $\mathcal{A}$-modules. Affine Darboux theorem. Rend. Circ. Mat. Palermo 58, 169-198 (2009)

17. Ntumba, P.P.: Cartan-Dieudonné Theorem for $\mathcal{A}$-Modules. Mediterr. J. Math. 7 (2010)

18. Ntumba, P.P., Orioha A.C.: Darboux theorem and fundamental geometries for $\mathcal{A}$-modules. (under refereeing) 